\title{
GaAs Quantum Cascade Lasers: Fundamentals and Performance
}

\author{
C. Sirtori \\ Thales Research and Technology, \\ 91404 Orsay Cedex, France \\ carlo.sirtori@thalesgroup.com
}

\begin{abstract}
Quantum engineering of the electronic energy levels and tailoring of the wavefunctions in $\mathrm{GaAs} / \mathrm{Al}_{x} \mathrm{Ga}_{1-x}$ As heterostructures allows to obtain the correct matrix elements and scattering rates which enable laser action between subbands. This article reviews the state-of-the-art of GaAs based quantum cascade lasers. These new light sources operate, with peak power in excess of $1 \mathrm{~W}$ at $77 \mathrm{~K}$, in the $8-13 \mu \mathrm{m}$ wavelength region, greatly extending the wavelength range of $\mathrm{GaAs}$ optoelectronic technology. Waveguides are based on an Al-free design with an appropriate doping profile which allows optical confinement, low losses and optimal heat dissipation. Finally, new active region designs aiming to improve the laser temperature dependence are discussed. Recent results on these devices confirm that the ratio between the conduction band discontinuity and the photon energy $\left(\Delta E_{\mathrm{c}} / E_{\text {laser }}\right)$ is the dominant parameter controlling their thermal characteristic. The maximum operating temperature of these devices is $280 \mathrm{~K}$ for lasers with emission wavelength at $\sim 11 \mu \mathrm{m}$.
\end{abstract}

Keywords: Semiconductor lasers, intersubband transitions, mid-infrared, optical gain, unipolar devices. 


\section{INTRODUCTION}

Quantum engineering of electronic energy states and wavefunctions using ultrathin layers $(0.5-100 \mathrm{~nm})$ of semiconductor compounds, with different compositions, allows the design and observation of quantum phenomena which are typically observed in atomic structures [1]. This approach is the basis for modifying, in an unique way, the optical and transport properties of semiconductors, opening avenues to artificial materials and the creation of useful devices.

The quantum cascade (QC) laser is an excellent example of how quantum engineering can be used to conceive efficient devices and emitters in the mid-infrared (IR). Since their first demonstration, QC lasers [2] have been devised to work in a wide range of wavelengths and temperatures $[3,4,5,6]$, by exploiting different designs, but always in the same material system: InP/GaInAs/AlInAs. Only recently the QC concepts were successfully implemented into a GaAs/AlGaAs heterostructure [7].

In this article we review the principal features of GaAs based QC lasers and give an overview on the performance which are attainable, at present, with this type of mid-IR semiconductor laser. In the first paragraph we illustrate the fundamentals of QC lasers and the advantage of having this technology available also on the GaAs industrial platform. The second paragraph focuses on the realisation of QC lasers and is divided into three parts: Active region design, Waveguide design and Laser fabrication. In the last paragraph we describe the most recent structures which have been designed to improve laser performance, giving special attention to increase the operating temperature.

\section{THE FUNDAMENTALS OF QUANTUM CASCADE LASERS}

Semiconductor diode lasers, including quantum well lasers, rely on transitions between energy bands in which conduction electrons and valence band holes are injected into the active layer through a forward-biased $p$ - $n$ junction and radiatively recombine across the material bandgap [8]. The latter essentially determines the emission wavelength. In addition, because the electron and hole populations are broadly distributed in the conduction and valence band according to Fermi's statistics, the resulting gain spectrum is quite broad and is of the order of the thermal energy.

The unipolar intersubband laser or QC laser differs in many fundamental ways from diode lasers. All the differences are consequences of two main features which are unique to quantum cascade lasers and distinguish them from conventional semiconductor light emitters: unipolarity (electrons only) and a cascading scheme (electron recycling). These two features, shown schematically in Figure 1, are independent, and can be used separately as it has been already demonstrated experimentally. In 1997 Garcia et al. [9] have demonstrated a cascade interband laser at $\sim 830 \mathrm{~nm}$, whereas Gmachl et al. [10] in 1998 have demonstrated an intersubband laser without a cascade scheme.

The unipolarity in QC lasers is a consequence of the optical transitions which occur between conduction band states (subbands) arising from size quantisation 


\section{UNIPOLARITY optical transition between subbands}
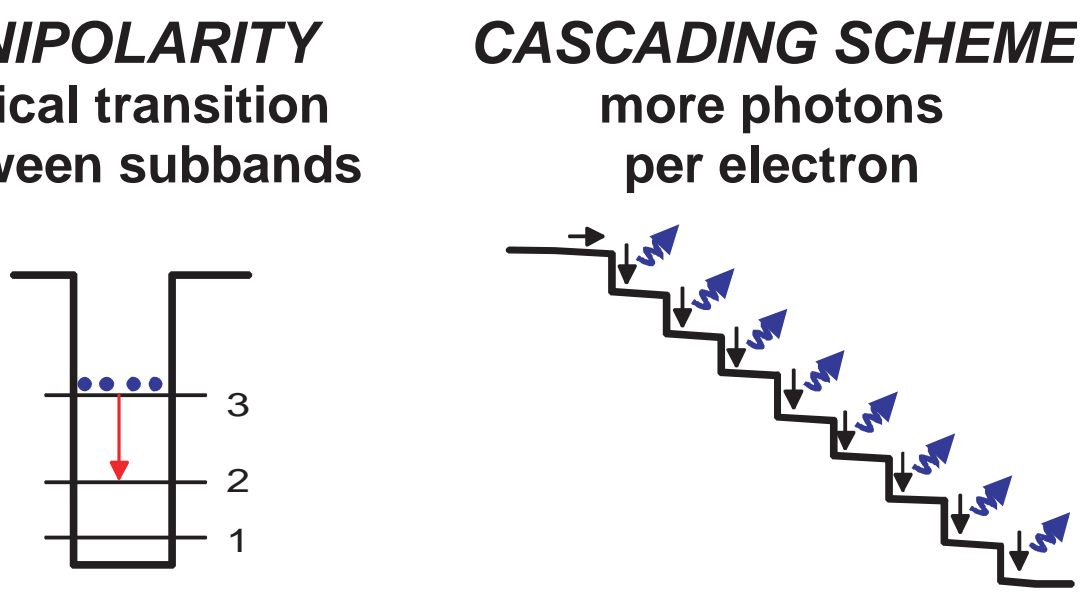

Figure 1. - Schematic representation of the two features that characterise a quantum cascade laser. Note that intersubband transition can be observed also for holes and therefore, QC lasers are also conceivable in valence band, for instance using SiGe quantum wells.

in semiconductor heterostructures. These transitions are commonly denoted as intersubband transitions. Their initial and final states are in the conduction band and therefore have the same curvature in the reciprocal space. If one neglects nonparabolicity, the joint density of states is very sharp, more similar to the case of atomic transitions. In contrast to interband transitions, the gain linewidth is now indirectly dependent on temperature through collision processes and many body effects. Moreover, for these devices the emission wavelength is not dependent on the band gap of constituent materials, but can be tuned by tailoring the layer thickness. The highest achievable photon energy is ultimately set by the constituent conduction-band discontinuity, while on the long wavelength side there are no fundamental limits preventing the fabrication of QC devices emitting in the far infrared.

The other fundamental feature of QC lasers is the multistage cascade scheme, where electrons are recycled from period to period, contributing each time to the gain and the photon emission. Thus each electron injected above threshold generates $N_{p}$ laser photons where $N_{p}$ is the number of stages, leading to a differential efficiency and therefore an optical power proportional to $N_{p}[11]$.

The first demonstration of a QC laser was achieved in 1994 at AT\&T (now Lucent Technologies) Bell Laboratories by Faist et al. [2] using an $\mathrm{Al}_{.48} \mathrm{In}_{.52} \mathrm{As} / \mathrm{Ga} .{ }_{47} \mathrm{In}_{.53}$ As/InP heterostructure. In the following years the Bell Labs group made a strong effort to improve the performance and illustrate the potential of these devices as a revolutionary light source for molecular spectroscopy [12,13]. Many important milestones for semiconductor lasers with emission wavelength in the mid infrared (3-15 $\mu \mathrm{m})$, such as room temperature operation and high cw output power $(\sim 1 \mathrm{~W})$ at cryogenic temperatures, were demonstrated for the first time using QC lasers [5,6,11]. However, for five years after its demonstration, all results were accomplished using 
a single semiconductor system: $\mathrm{Al}_{.48} \mathrm{In}_{.52} \mathrm{As} / \mathrm{Ga} .{ }_{47} \mathrm{In}_{.53} \mathrm{As} / \mathrm{InP}$. This is somehow in contradiction with the principle of these new devices, which, based on intersubband transitions, are essentially independent from the specific semiconductor system used.

The first demonstration of QC lasers in a different heterostructure was achieved in 1998 using $\mathrm{GaAs} / \mathrm{Al}_{0.33} \mathrm{Ga}_{0.67} \mathrm{As}$ and proved that the fundamentals of these devices are truly not bound to a particular material system [7]. Moreover the use of (Al)GaAs based heterostructures, which are the most widespread and developed among compound semiconductors, confers an additional technological value to these devices.

At present GaAs QC lasers have not yet reached the performance of the InP based devices, but have already shined light on some advantages which are attainable with a GaAs based technology. Among these, very significantly, is the utilisation of mature processing techniques, which are more suitable on GaAs than on InP based device. It is known, in fact, that standard dry etching techniques, like RIE or CAIBE, become more difficult to use when In compounds are present. In fact, the $\mathrm{InCl}_{3}$ reaction products are non-volatile at room temperature (note: $\mathrm{InCl}_{3}$ sublimates at $\sim 200{ }^{\circ} \mathrm{C}$ ), slowing down the etch rate and making the exposed surface very rough.

\section{DESIGN AND OPERATION OF A GaAs/AlGaAs QUANTUM CASCADE LASERS}

Although the overall principles accounting for population inversion are basically the same as for QC lasers grown on InP substrates, the use of $\mathrm{GaAs} / \mathrm{Al}_{x} \mathrm{G}_{1-x}$ aAs imposes significant modifications to the active region design, the waveguide, and device fabrication.

\subsection{Active Region Design}

Figure 2 shows the band diagram and the relevant wavefunctions of the first GaAs QC laser [7]. The barriers are obtained using $\mathrm{Al}_{x} \mathrm{Ga}_{1-x} \mathrm{As}$ with $x=0.33$. With this Al concentration, the conduction band discontinuity at the $\Gamma$ point is $\Delta E_{\mathrm{c}} \cong 295 \mathrm{meV}$ and the $\mathrm{AlGaAs}$ is a direct gap semiconductor with the $\mathrm{X}$ minima about $60 \mathrm{meV}$ above the $\Gamma$ valley (i.e., $355=\left(\Delta E_{\mathrm{c}}+60\right) \mathrm{meV}$ above that of GaAs) [14].

The active region design is based on a "anticrossed diagonal" scheme, where the wavefunction of the excited state of the laser transition $(n=3)$ has a reduced overlap with the lower state $(n=2)$ [15]. The spatial separation between the wavefunctions is tailored so that it decreases the electron-optical phonon scattering matrix elements while preventing a strong reduction of the dipole matrix element $z_{32}(1.6 \mathrm{~nm})$. The corresponding laser transition energy is $E_{32}=134 \mathrm{meV}$, but can be easily varied if a different emission wavelength is desired. The energy separation between state $n=1$ and $n=2$ is instead constant and tuned approximately equal to that of a longitudinal optical (LO) phonon (36 meV for GaAs). The calculated lifetime, due to the electron-optical phonon interaction [16], for the $n=3$ state is $\tau_{3}=1.5 \mathrm{ps}$, a factor 2 longer than a QC structure at same wavelength based on vertical transition. Our calculations also show that the population inversion condition $\tau_{32}-\tau_{2}>0$ is well satisfied, here $\tau_{32}=2.4 \mathrm{ps}$ is the relaxation time from the $n=3$ state into the $n=2$ 


\section{$\mathrm{GaAs} / \mathrm{Al}_{0.33} \mathrm{Ga}_{0.67} \mathrm{As}$}

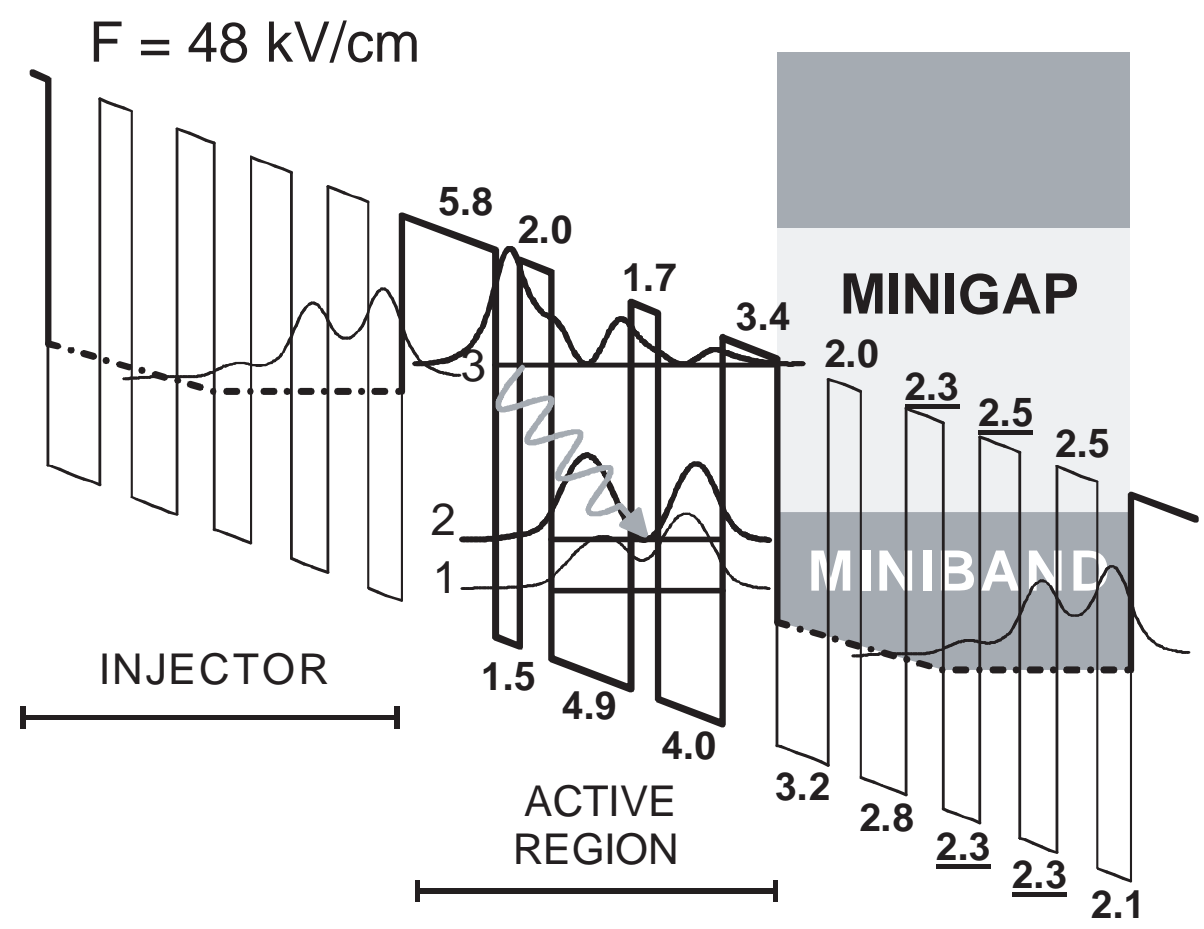

Figure 2. - Schematic conduction band diagram of a portion of the laser heterostructure at threshold bias. The thicknesses of the layers are indicated in $\mathrm{nm}$, the underlined numbers denote the four layers which are $n$-doped (sheet density $3.9 \times 10^{11} \mathrm{~cm}^{-2}$ per period). The wavy arrow indicates the transition $3 \rightarrow 2$ responsible for the laser action. The solid curves represent the moduli squared of the relevant wavefunctions. The calculated energy level differences are $E_{32}=134 \mathrm{meV}$ and $E_{21}=38 \mathrm{meV}$. The dash-dotted line in the injector represents the effective conduction band edges of the digitally graded region. Note the reduced spatial overlap between the $n=3$ and $n=2$ wavefunctions, a typical feature of laser based on diagonal transition.

state and $\tau_{2}=0.3 \mathrm{ps}$ is the life time of the $n=2$ state. The efficient carrier evacuation from the $n=2$ state is provided by resonant phonon emission of electrons that relax into the $n=1$ state and by electron escape via direct tunnelling into the injector of the adjacent period. Tunnelling escape is maximised by anticrossing the state $n=1$ and state $n=2$, so that its wavefunctions are strongly delocalised and can penetrate into the escape barrier, thus increasing their probability of tunnelling with states in the injector. 


\subsection{Waveguide Design}

Waveguide design of semiconductor lasers emitting in the mid/far-infrared $(4-20 \mu \mathrm{m})$ has to contend with much higher internal cavity losses and much thicker cladding layers than near-IR diode lasers $[17,18,19]$. For wavelengths $\geq 10 \mu \mathrm{m}$, the cladding thickness is at the limit of epitaxial growth.

Optical losses can reach very high values $\left(>100 \mathrm{~cm}^{-1}\right)$ and are at present one of the major limiting factors on the performance of mid-IR lasers $[17,18]$. This problem is more severe for low gap semiconductor lasers [19] than for QC lasers. For the latter $\alpha_{W}$ is predominantly controlled by free carrier absorption, whereas for the former there is also a strong contribution from intraband absorption associated with subband resonances, especially in the valence band [19]. Both these effects increase with the carrier concentration and are therefore very sensitive to the thermally activated population across the band gap. This is a fundamental advantage of QC lasers which are based on high gap semiconductors, where the thermally activated population at room temperature is completely negligible. This is obviously not the case for lasers based on narrow-gap semiconductors, for which the thermal population at room temperature can be a significant fraction of the carriers at threshold. This important difference on the temperature dependence of the waveguide losses could be a major asset for QC laser technology.

In recent GaAs QC lasers the planar optical confinement relies on a novel Alfree design [20]. In these waveguides the variations of the refractive index are exclusively based on a change in the doping concentration rather than on alternating materials. Apart from the lower losses, which will be discussed in detail later, there are several advantages in fabricating mid-IR waveguides without AlGaAs cladding layers. In these layers the ionised carrier concentrations have significant variations as a function of different environmental conditions (typically, stray light illumination and temperature). These variations produce strong instabilities on device thresholds and slope efficiencies as it has been already reported [21]. In addition, the growth of thick, high-quality GaAs layers is much easier than AlGaAs layers, which are anyway limited to a maximum thickness of approximately $1.5 \mu \mathrm{m}$, due to residual strain. Also, the ternary alloys, such as AlGaAs, have very poor thermal conductivity compared with the binary crystals. Simple calculation shows that by using GaAs cladding layers, the thermal resistance of the lasers is reduced by a factor 2. Finally, we eliminate any heterobarriers between the contacts and the active region that may increase the differential resistance across the device.

Figure 3 presents all the relevant parameters and the resulting mode profile of the waveguide we are using. The active region is sandwiched between two thick layers $(4.5 \mu \mathrm{m})$ of GaAs, no AlGaAs is used as cladding material. In our waveguides the decrease of the refractive index, needed for planar optical confinement, is achieved by increasing the doping concentration in the last micron of the GaAs layers (Fig. 3). The doping $\left(6 \times 10^{18} \mathrm{~cm}^{-3}\right)$ is chosen so to shift the plasma frequency of the $n^{++}$ layers close to that of the laser [22]. This strongly depresses the real part of the refractive index, $n$ (middle part of Fig. 3), but also increases its imaginary part, $k$, thus the absorption coefficient of these layers (lower part of Fig. 3) [22]. To avoid very high waveguide losses, it is necessary to minimise the overlap factor between 


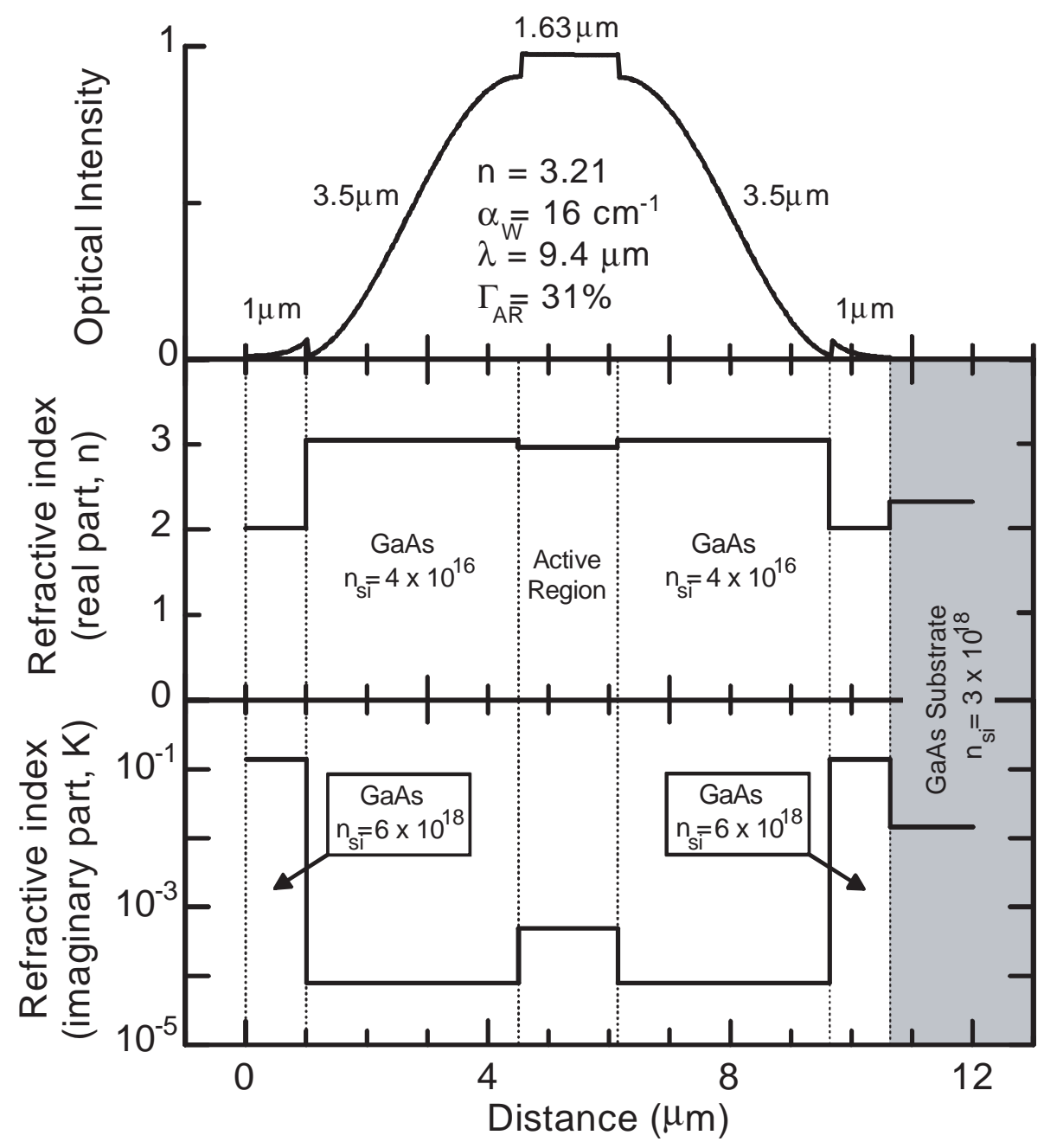

Figure 3. - (Upper part) Calculated intensity distribution of the fundamental TM mode of the waveguide. Also indicated are the relevant thicknesses of the structure and the important parameters characterising the waveguide. (Middle part) Real part of the refractive index profile in the direction perpendicular to the layers. (Lower part) Imaginary part of the refractive index profile in the direction perpendicular to the layers. Note that the latter increases more than three orders of magnitude in plasmon confinement cladding layers.

the $n^{++}$layers and the optical mode. This is readily achieved by separating these layers from the active region by two thick low doped $\left(4 \times 10^{16} \mathrm{~cm}^{-3}\right)$ GaAs layers. The calculated overlap factor with the lossy cladding layers, $\Gamma_{n^{++}}$, is only 0.008 , but it accounts for $90 \%$ of the total waveguide losses. In fact, in these layers the absorption coefficient imposed by free carriers [23], at $\sim 9.5 \mu \mathrm{m}$ wavelength, $\alpha_{n^{++}}$, is $1740 \mathrm{~cm}^{-1}$, 


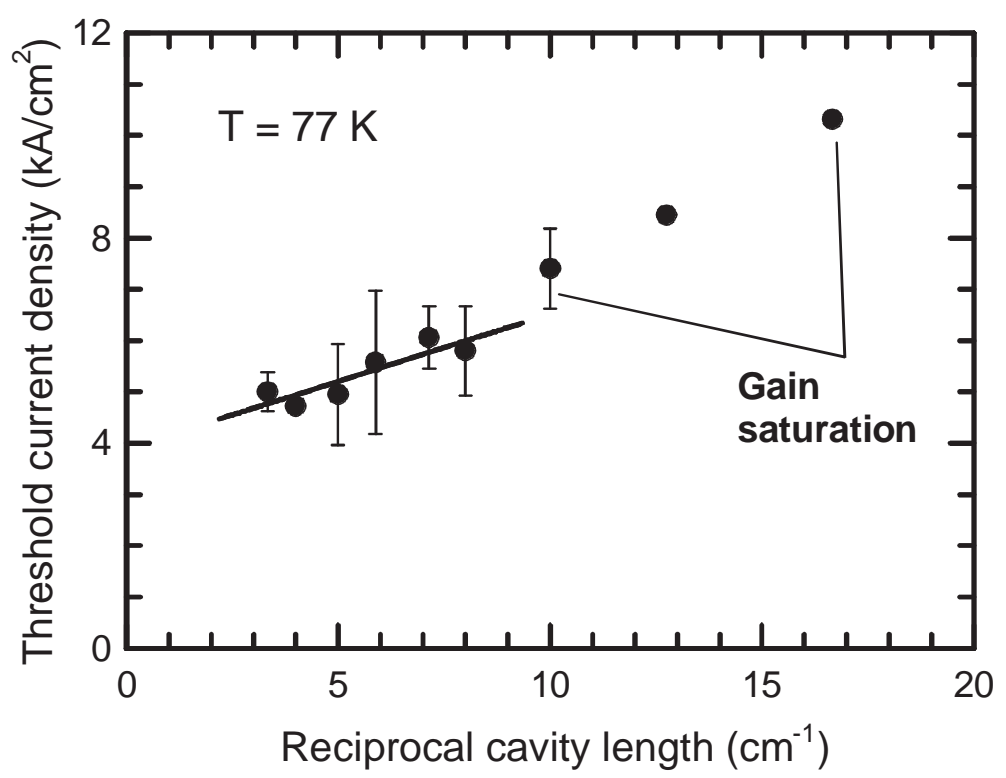

Figure 4. - Measured threshold current density versus reciprocal cavity length at $77 \mathrm{~K}$. For current densities above $7 \mathrm{kA} \cdot \mathrm{cm}^{-2}$ the data show evidence of gain saturation. The solid line is the least square fit to the data below saturation. From its slope and intercept the modal gain and the waveguide loss are determined. For this measurement 35 devices were tested.

which multiplied by $\Gamma_{n^{++}}$gives $14 \mathrm{~cm}^{-1}$. Note that these waveguides are not based on a pseudo-metallic optical confinement, since the real part of the complex dielectric function of the heavily doped layers is still the dominant $(n \gg k)$ [20].

The waveguide losses, $\alpha_{W}$, in QC lasers are typically characterised using two independent methods: one based on a plot of the threshold current density versus reciprocal cavity length $(1 / L)$ and the other on an analysis of subthreshold emission spectra for different injected currents.

The former method is based on the threshold condition which can be written as:

$$
J_{\text {th }}=\frac{\alpha_{W}+\alpha_{M}}{g \Gamma_{A R}}=\frac{\alpha_{W}}{g \Gamma_{A R}}-\frac{\ln (R)}{g \Gamma_{A R}} \frac{1}{L}
$$

where $\alpha_{M}=-\ln (R) / L$ is the mirror loss, $R$ is the facet reflectivity $(=0.27)$ and $g$ is the gain coefficient. Figure 4 shows the plot of the measured threshold versus $1 / L$ at $77 \mathrm{~K}$ for characteristic laser with an Al-free waveguide and emission wavelength at $\sim 9 \mu \mathrm{m}$. The data can be correctly fitted with equation (1) only up to current densities of the order of 7-8 kA.cm ${ }^{-2}$; above this value they lose their linear dependence, a sign of gain saturation. From the slope and the intercept of the straight line below the saturation point, one gets $\alpha_{W}$ and the modal gain coefficient $g \Gamma_{A R}$. For the latter, we find $4.9 \mathrm{~cm} \cdot \mathrm{kA}^{-1}$ and the corresponding value for the waveguide losses is $19 \mathrm{~cm}^{-1}$.

The second method is based on a measurement of the fringe contrast of the 


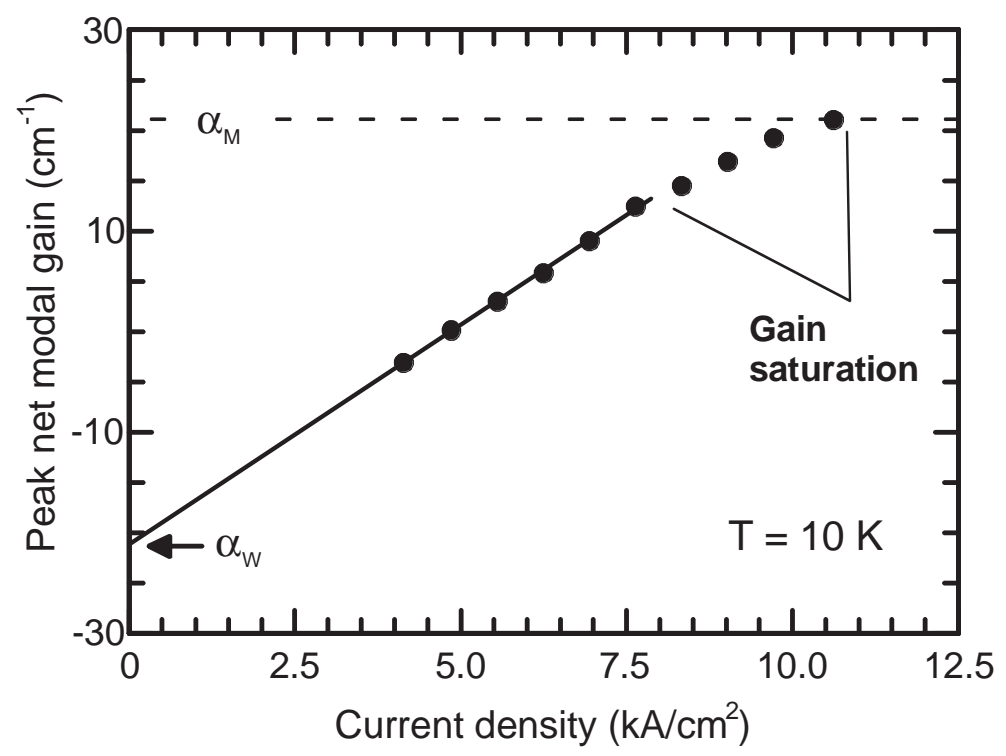

Figure 5. - Net peak modal gain $G_{M}\left(\lambda_{\text {peak }}\right)-\alpha_{W}$ of a $0.6 \mathrm{~mm}$-long laser as deduced from the subthreshold emission spectra plotted as a function of the current density $J$. It is apparent that gain saturation is occurring above $7.5 \mathrm{kA} . \mathrm{cm}^{-2}$, in agreement with the data of Figure 3. The laser threshold is reached when $G_{M}\left(\lambda_{\text {peak }}\right)-\alpha_{W}$ exactly compensate the mirror losses $\alpha_{M}=21 \mathrm{~cm}^{-1}$ (dashed line). The slope and intercept of the line fit to the data, prior to saturation, give $G_{M}$ and $\alpha_{W}$.

Fabry-Perot modes of the cavity below threshold. The net modal gain $\left(G_{M}(\lambda)-\alpha_{W}\right.$, with $G_{M}(\lambda)=J g(\lambda) \Gamma_{A R}$, where $J$ is the current density and $g(\lambda)$ the gain coefficient as a function of the wavelength) is extracted from the fringe contrast using numerical Fourier analysis of the subthreshold spectra in a modified version of the Hakki-Paoli technique [24]. In Figure 5 the peak net modal gain $\left(=J g\left(\lambda_{\text {peak }}\right) \Gamma_{A R}-\alpha_{W}\right)$ is plotted versus drive current density. We can still observe the linear dependence up to $7.5 \mathrm{kA} . \mathrm{cm}^{-2}$. After this value the gain saturates, in accordance with the previous measurement. From the data in the linear regime, we derive $g \Gamma_{A R}=4.4 \mathrm{~cm} \cdot \mathrm{kA}^{-1}$ and $\alpha_{W}=21 \mathrm{~cm}^{-1}$ in good agreement with the $1 / L$ method.

The measured values of the waveguide losses are also in good agreement with the predicted value of $16 \mathrm{~cm}^{-1}$, which is calculated by solely taking into account the free carrier absorption of the different layers. This value is approximately half of the previously measured internal losses in AlInAs/InGaAs/InP waveguides in the same wavelength range. This is consistent with the assumption that free carrier absorption is the dominant factor in the waveguide losses, since a higher effective mass corresponds a lower absorption $\left(m_{\mathrm{GaAs}}^{*}=0.067 m_{0} ; m_{\mathrm{GaInAs}}^{*}=0.0427 m_{0}\right)$. By optimising the doping in the $n^{++}$layers we were able to obtain structures with $\alpha_{W}=15 \mathrm{~cm}^{-1}$. The light vs. current characteristics $(L-I)$ of these devices operating in pulsed mode at $77 \mathrm{~K}$ is shown in Figure 6. Note that the power rises up to $1.5 \mathrm{~W}$, 


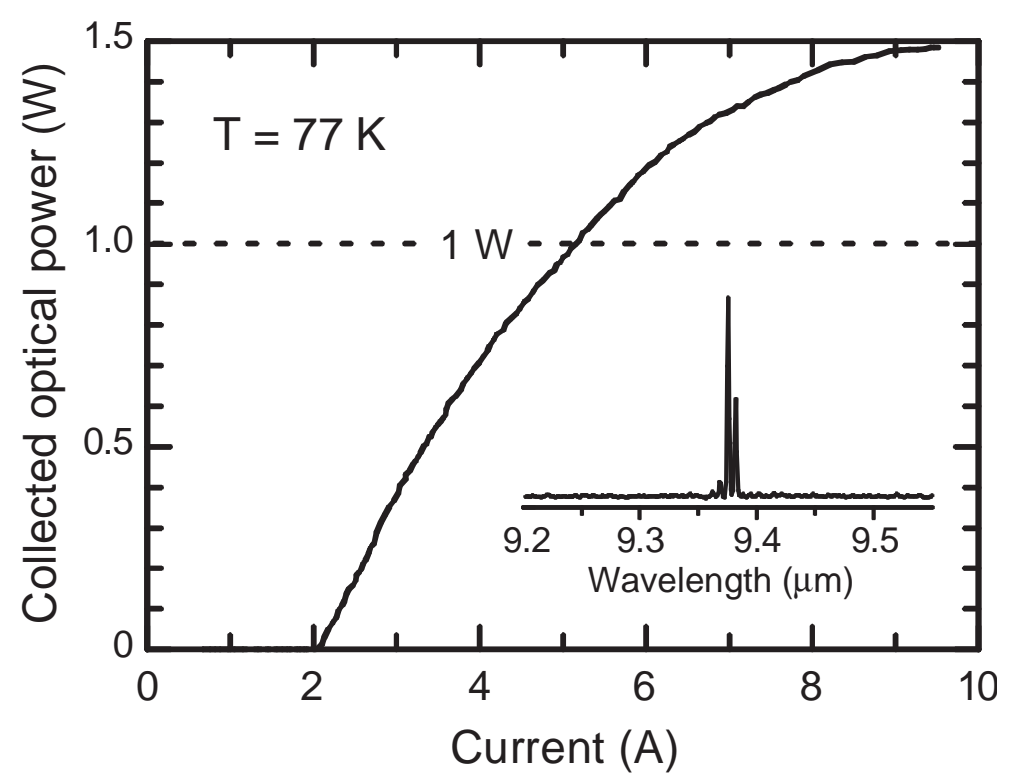

Figure 6. - Light-current $(L-I)$ characteristic, at $77 \mathrm{~K}$, as recorded using a $f / 0.8$ optics and a calibrated room-temperature $\mathrm{HgCdTe}$ detector from a single facet with approximately $60 \%$ collection efficiency. The device ( $2 \mathrm{~mm}$ long, $30 \mu \mathrm{m}$ wide) is driven in pulsed mode (100 $\mathrm{ns}$ width, $1 \mathrm{kHz}$ repetition rate), with the measurement performed using an adjustable gate integrator. The high resolution pulsed spectrum of this device is shown in the inset.

the highest peak power ever reported for a laser with emission wavelength above $5 \mu \mathrm{m}$.

\subsection{Laser Fabrication}

Samples are processed into mesa etched ridge waveguides by optical contact lithography and deep wet chemical etching. Typical etching rates of the acids are in the range of $600 \mathrm{~nm} / \mathrm{min}$. After thinning down the substrate to $100 \mu \mathrm{m}$, a standard $\mathrm{AuGe} / \mathrm{Ni} / \mathrm{Au}$ contact is evaporated on the backside and alloyed at $400{ }^{\circ} \mathrm{C}$ for one minute. For the insulation of the ridges a $300-\mathrm{nm}$-thick $\mathrm{Si}_{3} \mathrm{~N}_{4}$ layer is deposited by a sputtering technique. Windows in the nitride are patterned on top of the ridges by reactive ion etching using a $\mathrm{SF}_{6}$ plasma. The top $\mathrm{AuGe} / \mathrm{Ni} / \mathrm{Au}$ contact is annealed in order to avoid parasitic series resistance and power dissipation.

Recently we have also developed an epi-side down technique which allows a much better dissipation of the heat generated inside the devices, see Figure 7. For lasers mounted in this way the power generated in the active region has to cross only the top cladding and when it reaches the copper mount is easily dissipated, since the latter has a thermal conductivity 100 times better than GaAs. This technique, widely exploited in high power diode laser technology, is directly applicable to unipolar semiconductor lasers. 


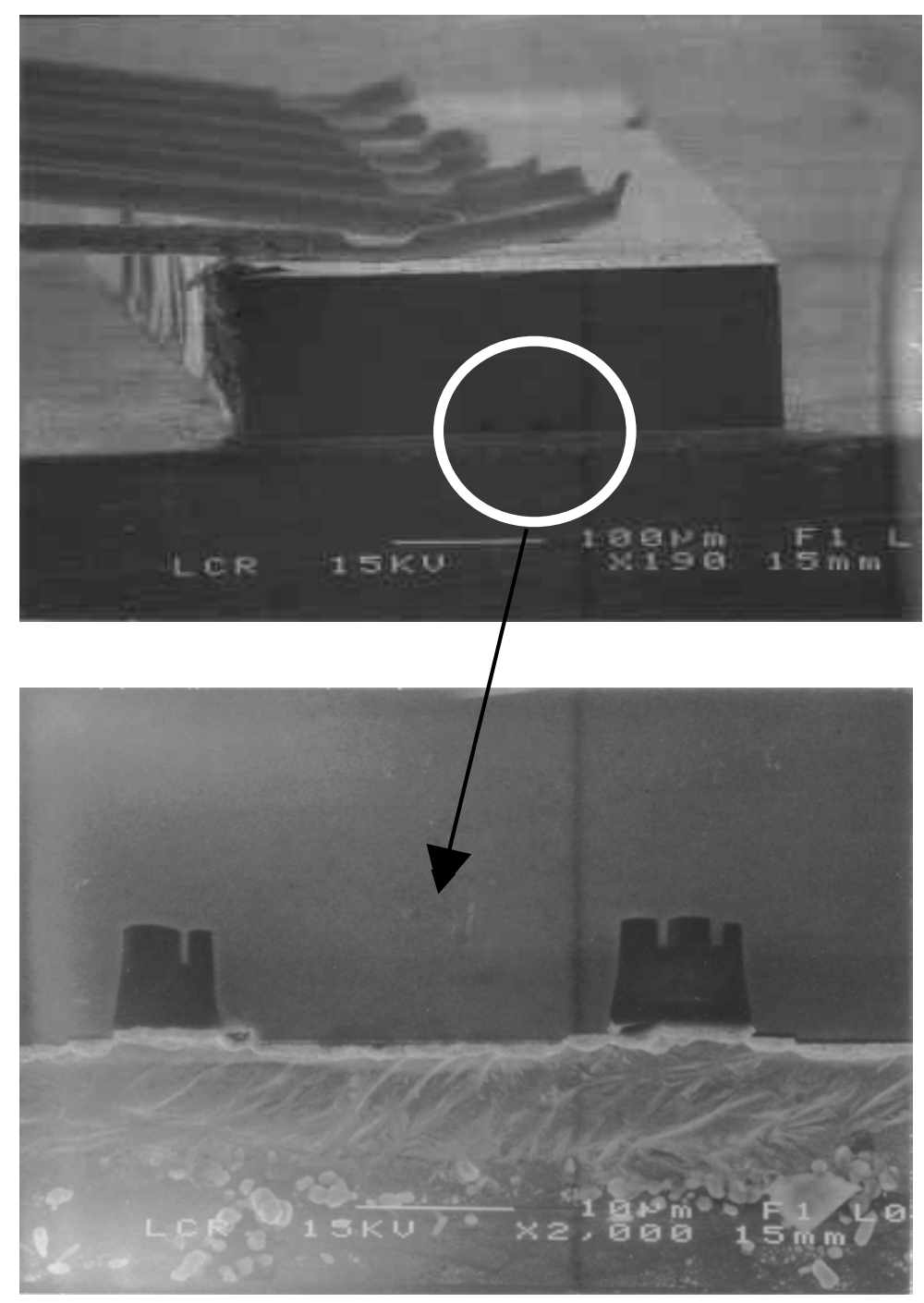

Figure 7. - STM image of a junction down device. Note that the epilayer is mounted close to the copper holder. Top panel: the whole device. Bottom panel: magnification of double trenches for the lateral confinement of the current and of the optical mode.

Also in this case, devices are processed into ridge lasers $(20-30 \mu \mathrm{m})$. The ridge are defined by dry etching deep trenches across the active region, which are then filled with polymer to planarise the device. A silicon dioxide layer is used as insulator to prevent short circuit outside the ridge. An $\mathrm{Au} / \mathrm{Ni} / \mathrm{GeAu}$ contact is finally evaporated on top the structure. The lasers are then cleaved in bars of 1-3 mm length and the laser facets are left uncoated. Finally samples are indium soldered epilayer down to 
$\mathrm{Cu}$ holders, wire bonded and mounted inside a cryostat or on a Peltier element. The junction down mounting has also allowed us to measure significant average power at $77 \mathrm{~K}$ (in the order of $30 \mathrm{~mW}$ ). This has been obtained driving the lasers with high duty cycles. For short pulses ( $100 \mathrm{~ns})$, no evidence of self heating has been seen up to $10 \%$. By exploiting dry etching techniques sophisticated GaAs QC lasers have been recently produced by the group lead by G. Strasser at the Technical University in Vienna. Among the most important results, it worth mentioning: complex coupled DFB lasers obtained by lithography and ion milling, tuneable coupled cavities and quadrupole microlasers $[25,26,27]$.

\section{NEW GaAs LASER STRUCTURES WITH IMPROVED THERMAL BEHAVIOUR}

The peak output-power obtained with GaAs lasers up to $77 \mathrm{~K}$ is comparable or better than that on InP/GaInAs/AlInAs lasers. However, GaAs lasers have threshold current densities, at cryogenic temperature in the $3-5 \mathrm{kA} . \mathrm{cm}^{-2}$ range, whereas the threshold in InP based lasers are already below $1 \mathrm{kA} \cdot \mathrm{cm}^{-2}$. The high threshold current densities are the main reason which prevents continuous wave $(\mathrm{cw})$ operation of GaAs based lasers, while for the other devices cw operation has been demonstrated up to $180 \mathrm{~K}$ [28].

Using a new active region design GaAs lasers have recently reached $260 \mathrm{~K}$ in pulsed operation [29], a very encouraging result, which puts room temperature operation a close and reachable goal.

Recent studies on the gain mechanism in GaAs lasers show a non-unity injection efficiency into the upper level of the laser transition [30,31]. This is due to the thermal activation of electrons from the injector directly into the continuum states (Fig. 8) and is the principal reason which hinders lower thresholds and room temperature operation. To suppress or at least reduce this parasitic current path, it is necessary to increase the energy separation between the injector and the continuum states $\Delta E_{\text {act }}$. Furthermore, band-structure calculations show that this escape probability increases with the applied electric field and gives rise to an even more pronounced injector/excited-continuum coupling [31].

Finally, scattering assisted injection of electrons into lateral valleys $(X, L)$ above the $\Gamma$-point of the GaAs and the AlGaAs alloy could also have an influence on device performance. All these mechanisms deteriorate the optical gain of the laser structure.

To suppress or at least reduce the parasitic current path, it is necessary to increase the energy separation between the injector/state $n=3$ and the continuum states $\Delta E_{\text {act }}$. This can be readily achieved in two different ways: the first is by increasing the wavelength of operation and therefore, sinking the excited level of the laser transition inside the quantum wells (Fig. 8); the second is to use higher conduction band offsets to deepen the levels inside the multiquantum well potential and hence have more freedom to increase the energy separation between the subbands (Fig. 10). Both these approaches have shown significant improvements of the thermal behaviour of our lasers, and will be discussed in details in the following two sections $[29,32]$. 


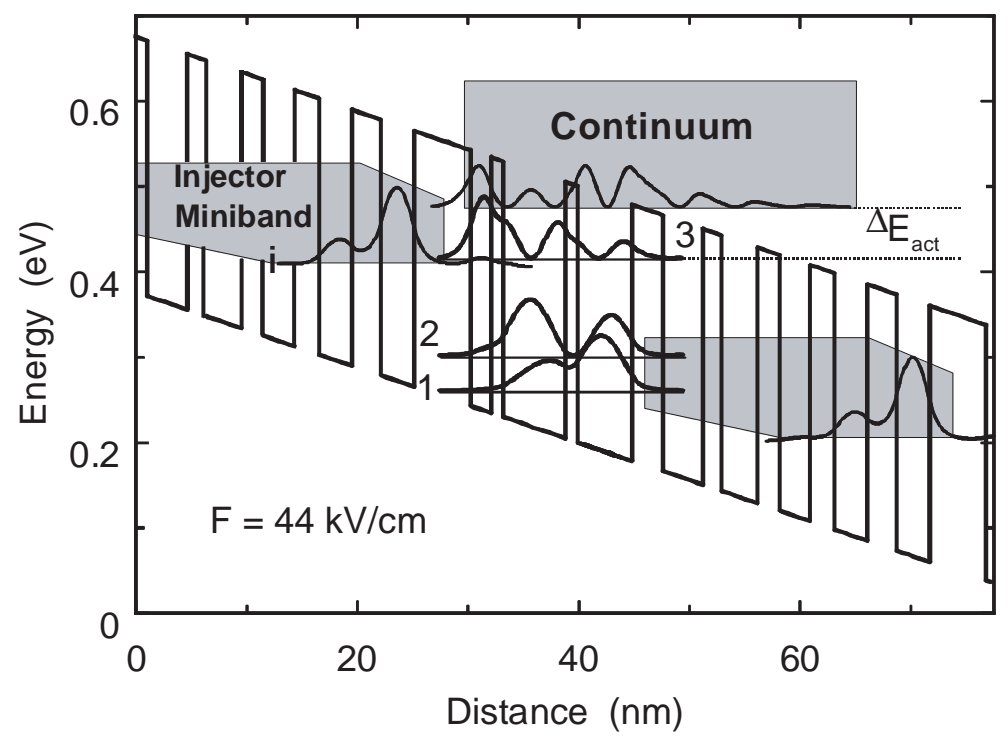

Figure 8. - Conduction band energy diagram of a portion of an $11 \mu \mathrm{m}$ quantum cascade laser under an applied electric field $F=44 \mathrm{kV} \cdot \mathrm{cm}^{-1}$. The subband alignment corresponds to the situation at threshold. Shown are the moduli squared of the relevant wavefunctions. The layer sequence of one period of structure, in nanometers, from left to right starting from the injection barrier is 51/19/11/56/11/49/28/36/17/32/20/28/22/30/26/30. $\quad \mathrm{Al}_{0.3} \mathrm{Ga}_{0.7}$ As layers are in bold. Underlined layers are $n$-doped with Silicon $N_{d}=8 \times 10^{17} \mathrm{~cm}^{-3}$.

\section{1. $\quad \mathrm{Al}_{.33} \mathrm{Ga}_{.67} \mathrm{As} / \mathrm{GaAs} 11 \mu \mathrm{m}$ Lasers}

The structure presented in this section aims to reduce the parasitic electron transfer while keeping the aluminium composition at 33\% and hence the conduction band discontinuity unchanged. The active region, which is shown in Figure 8, consists of three coupled quantum wells based on the "diagonal anticrossed" scheme, which has been commented in Section 1. The lifetime of the upper laser level $n=3$ is $\tau_{3}=\left(\tau_{31}^{-1}+\tau_{32}^{-1}\right)^{-1}=1.2 \mathrm{ps}\left(\tau_{32}=2 \mathrm{ps}\right)$. The optical matrix element is $z_{32}=2.1 \mathrm{~nm}$. Under an external field of $F=44 \mathrm{kV} \cdot \mathrm{cm}^{-1}$, we calculate an energy difference $E_{32}=$ $112 \mathrm{meV}(\lambda=11.1 \mu \mathrm{m})$. The following features characterise the essence of the design of the active region. In comparison with previous QC lasers, we have increased the thickness of each of the three strongly coupled quantum wells of the active region. This lowers the laser transition energy (3-2) and allows a better confinement of the $n=3$ state. At the same time the energy difference of the two anticrossed states $n=1$ and $n=2$ is kept close to the LO phonon energy to assure fast depopulation of the state $n=2$. The necessary energy splitting is achieved by reducing the width of the barrier that couples the two thick wells of the active region. Under typical biasing conditions the energy separation, $\Delta E_{\text {act}}$, between the injector/excited states and miniband-like continuum states has been increased. With the $n=3$ state as a reference point, 


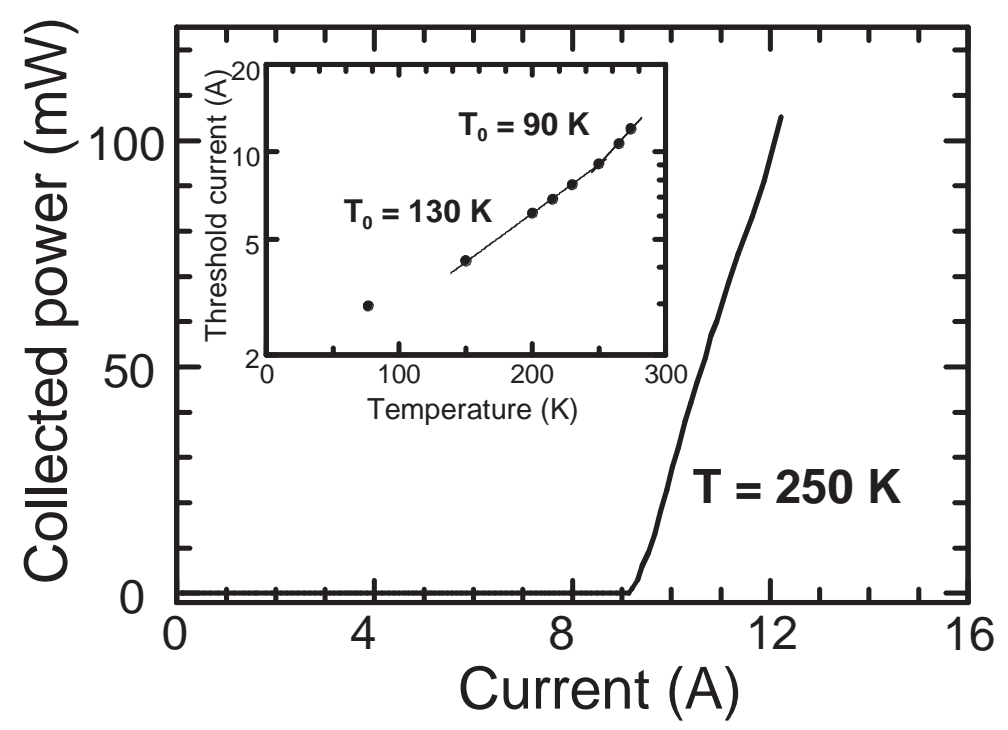

Figure 9. - Collected peak optical power from a single facet of an $11 \mu \mathrm{m}$ quantum cascade laser vs. drive current at $250 \mathrm{~K}$ heat sink temperature. Current pulses are $60 \mathrm{~ns}$ long, injected at $1-10 \mathrm{kHz}$ repetition rate. The calculated collection efficiency of the apparatus is $60 \%$. Inset: temperature dependence of the threshold current density. The maximum operating temperature is $280 \mathrm{~K}$.

bandstructure calculations give an energy difference of $\Delta E_{\text {act }}=58 \mathrm{meV}$ to the lowest delocalised continuum state. This value is significantly higher than the one obtained from a comparative calculation for our $\lambda \approx 9 \mu \mathrm{m}$ structure [7], $\Delta E_{\text {act }}=38 \mathrm{meV}$, under appropriate biasing conditions (close to resonance). As a consequence, the thermally activated leakage of electrons to the continuum should be significantly reduced in this laser structure. We believe that this design feature notably improves the high temperature operation of the laser.

The pulsed $L-I$ characteristics of a representative laser operating at $250 \mathrm{~K}$ is reported in Figure 9. The collected output power is in excess of $100 \mathrm{~mW}$ and the maximum operating temperature have recently exceeded our previous published data [29], and reached $280 \mathrm{~K}$. The threshold current density as a function of the temperature (inset of Fig. 9) follows the typical exponential dependence $\left(\exp \left(T / T_{0}\right)\right)$ with $T_{0}=130 \mathrm{~K}$ up to $250 \mathrm{~K}$ and $T_{0}=90$ thereafter. The estimated waveguide losses in these devices have been measured using the modified Hakki-Paoli method and are $44 \mathrm{~cm}^{-1}$. This rather high value is a consequence of the non-optimised waveguide design, but also of the longer emission wavelength which is always leads to higher losses. 


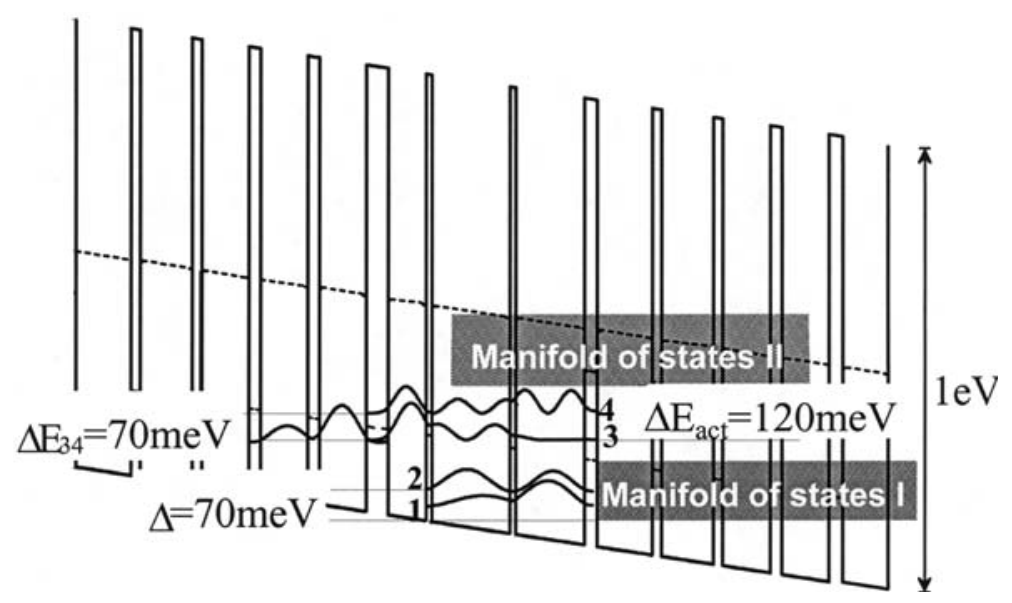

Figure 10. - Conduction band diagram of an injector/active region/injector sequence of a AlAs/GaAs quantum cascade laser with emission wavelength at $11 \mu \mathrm{m}$. Note that the manifold of states II is equivalent to the continuum states, located above the barriers of the active region in Figure 8. Here the height of the AlAs barriers permits the confinement of these states and therefore to increase their energy separation $\Delta E_{\text {act }}$. The layer sequence of one period of the structure, in nanometers, from left to right starting from the injector barrier is 1.9/3.3/0.5/6.8/0.5/6.0/1.1/4.8/0.8/4.5/0.8/4.1/1.0/4.1/1.0/4.1. AlAs layers are in bold. The underline layer is $n$ doped. The dashed line represents the $X$-valley conduction band profile.

\subsection{AlAs/GaAs Lasers}

As mentioned above, another solution to increase the energy separation between the injector and the continuum states, located above the $n=3$ state, is to increase the barrier height, therefore their Al content. To this end we used AlAs as barrier material to fully exploit the $\Gamma$ point conduction band discontinuity of $\sim 1 \mathrm{eV}$ with respect to $\mathrm{GaAs}$. The effective band offset in the AlAs/GaAs heterostructure is obviously much lower and is determined by the $\Gamma-X$ indirect discontinuity of $\sim 195 \mathrm{meV}$. Nevertheless, this does not influence the performance of our devices since the AlAs layers are much thinner than the GaAs ones and therefore the quantum confinement pushes the ground state of the $X$-valley-quantum-well almost to the level of the GaAs- $X$ minimum. Moreover the GaAs layers act as thick tunnelling barriers which hinder the perpendicular transport in $X$ valley. Consequently, electrons scattered into the $X$ valleys do not contribute to the transport and cannot directly influence the optical gain.

In view of these considerations and the results we are going to present it is safe to state that $\mathrm{QC}$ active regions in the $\mathrm{AlAs} / \mathrm{GaAs}$ heterostructure can be designed by taking into account only the $\Gamma$ discontinuity if the ratio $\mathrm{Al} / \mathrm{Ga} \leq 0.3(\mathrm{Al} / \mathrm{Ga}=0.17$ in our structure).

In the $\mathrm{AlAs} / \mathrm{GaAs}$ active regions (Fig. 10), the possible thermal activated processes are practically suppressed. The $n=4$ state is more than $70 \mathrm{meV}$ above the excited 


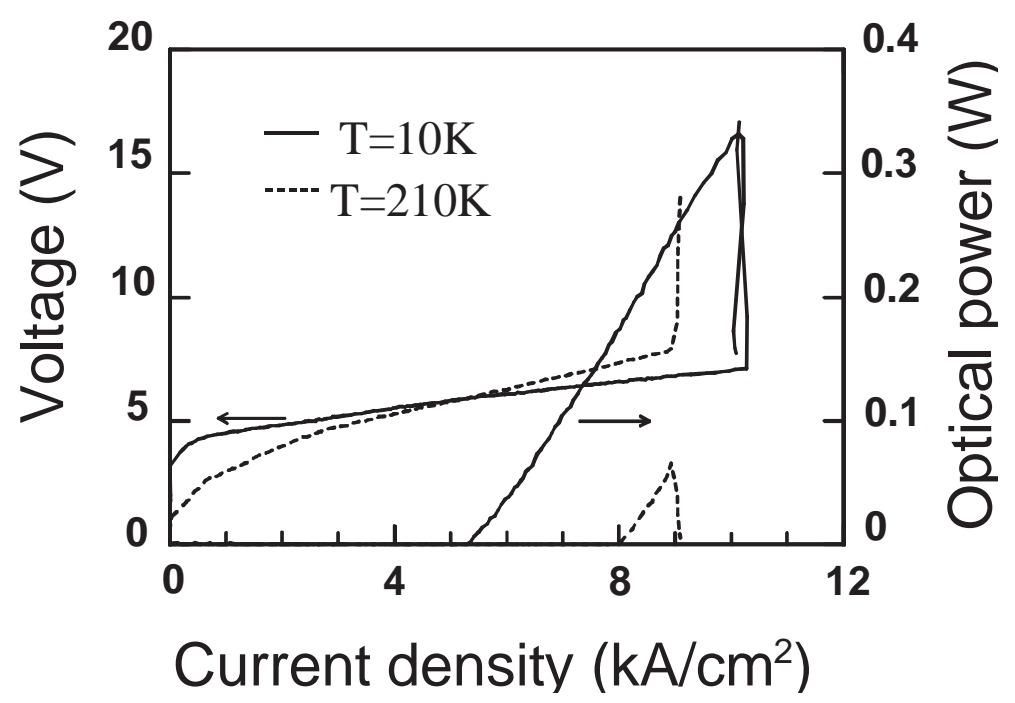

Figure 11. - Voltage and output power against current density of a 3-mm-long and 20- $\mu \mathrm{m}$ wide device, for two different operating temperatures (solid lines $T=10 \mathrm{~K}$, dashed lines $T=210 \mathrm{~K}$ ). Devices were driven with $100 \mathrm{~ns}$ pulses at $1 \mathrm{kHz}$ repetition frequency. The laser output from a single facet is collected with an $f / 0.8$ optical system.

state of the laser transition. This state is also very well confined within the three wells which define the active region and would be unlikely to contribute to the transport. In addition, the bottom of the manifold of extended states II [33], which we believe is the main channel for the thermally activated escape of electrons, is situated $120 \mathrm{meV}$ above the state $n=3$ ( $\Delta E_{\text {act }}$ in Fig. 10). In QC lasers, this quasi-miniband is usually placed at roughly the same energy as the $n=4$ state, which therefore becomes the coupling state between the injector and the extended states responsible for carrier leakage. Finally, as in previous QC lasers (based on GaAs or InP substrate), we avoid electron thermal back-filling into the $n=2$ state from the injector ground state, by separating these states of an energy $\Delta \geq 70 \mathrm{meV}$.

The electron lifetime on excited subbands is controlled by the electron-longitudinal optical phonon interaction. Due to the large elastic discontinuity between the two materials the AlAs/GaAs heterostructure cannot be correctly described using a model for bulk phonons. The scattering rates of the subbands $n=1, n=2$, and $n=3$, which permit an estimation of the population inversion, were calculated within the framework of the macroscopic dielectric model [34]. Our calculations show that, taking into account both confined and interface phonons, the relaxation time from the $n=3$ state into $n=2$ state is $\tau_{32}=1.6 \mathrm{ps}$ and from $n=3$ into $n=1$ is $\tau_{31}=4.5 \mathrm{ps}$.

The collected peak power from a single facet versus injected current densities, at two different heat sink temperatures, is displayed in Figure 11, for a 3-mm-long device. At $10 \mathrm{~K}$ the threshold current density is $5.2 \mathrm{kA} \cdot \mathrm{cm}^{-2}$ and the maximum peak power is in excess of $350 \mathrm{~mW}$, limited by the occurrence of negative differential 


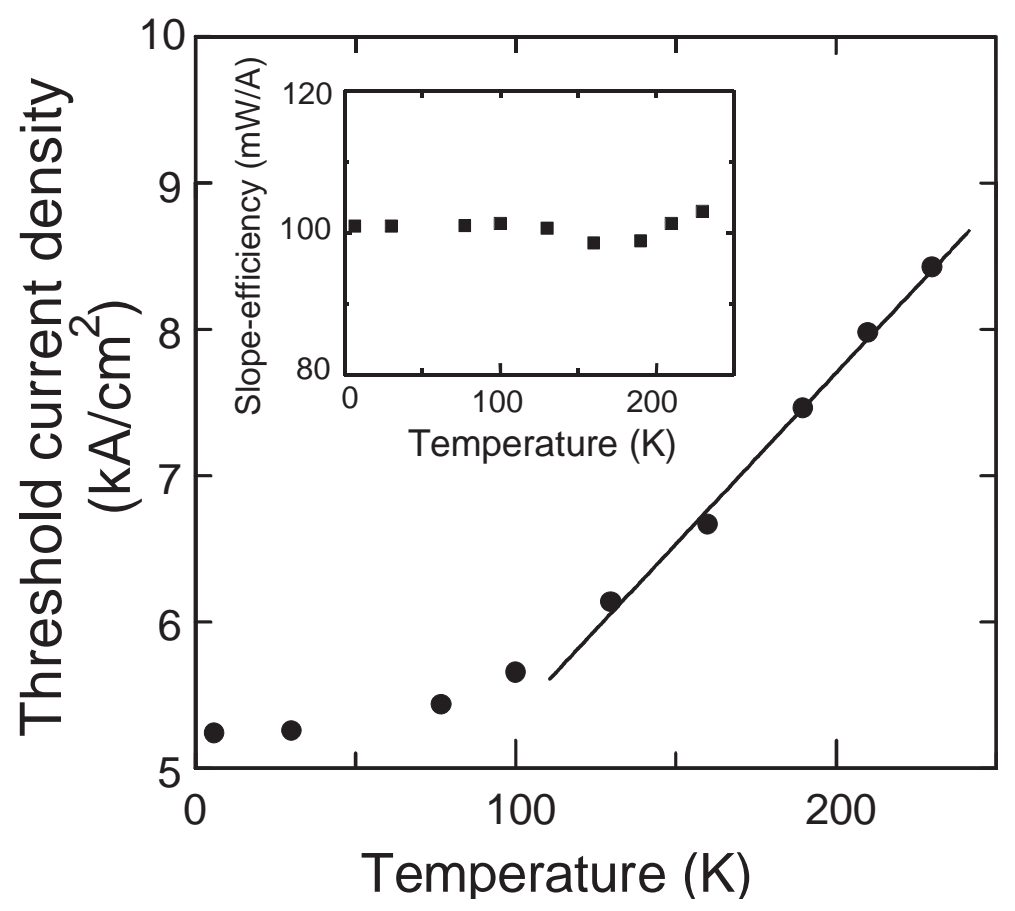

Figure 12. - Threshold current density as a function of temperature for a 3-mm-long and 20$\mu \mathrm{m}$-wide device. The solid line shows the range over which $J_{\text {th }}$ scales linearly (130-230 K). Note that an exponential fit of the data on the same range would give a characteristic temperature $T_{0}=320 \mathrm{~K}$. Inset: slope efficiency against temperature for the same device.

resistance (NDR) at an applied bias of $8 \mathrm{~V}$. The temperature dependence of the threshold current density does not follow the usual exponential behaviour. As can be seen in Figure 12, between 130 and $230 \mathrm{~K}$ the data have a linear dependence which is stressed by the straight line on the graph. However, an exponential fit of the data over this range would give a characteristic temperature $T_{0}=320 \mathrm{~K}$. In addition the differential slope efficiency is nearly constant over the whole temperature range of operation (inset Fig. 12). This is also evidence for the suppression of thermally activated leakage.

In this structure, laser action at temperatures higher than $230 \mathrm{~K}$ is prevented by the occurrence of NDR when the voltage reaches $8 \mathrm{~V}$ (Fig. 11). This is due to the breaking of the alignment between the injector ground state and the excited state of the laser transition, which dramatically reduces the amount of carriers entering into the active region. In addition, the differential resistance of the device increases with temperature, thus reducing the current density needed to reach the limiting voltage. The available current range before the occurrence of the NDR is therefore reduced when the temperature is increased. 


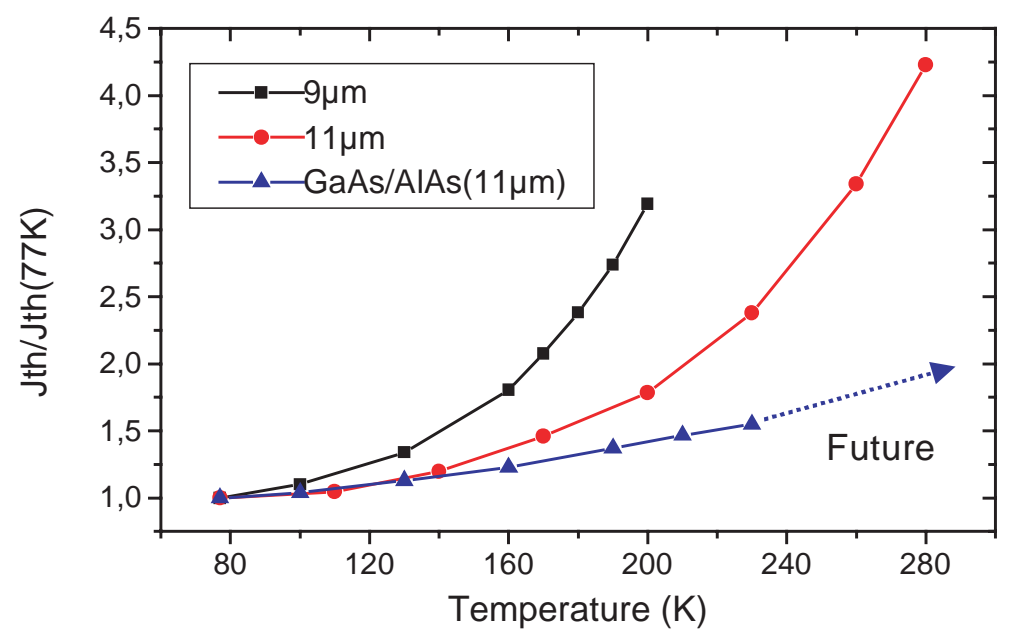

Figure 13. - Comparison of the relative temperature dependence of the threshold current density for the devices discussed in the text. The improvements in the temperature dependence and maximum operating temperature arise from successively increasing the lasing wavelength and the $\Delta E_{\text {act }}$. Squares show a typical characteristic for an $\mathrm{Al}=33 \%$ based heterostructure emitting $\sim 9 \mu \mathrm{m}$, with maximum operating temperature limited to $200 \mathrm{~K}$. The circles are for a similar heterostructure emitting at $\sim 11 \mu \mathrm{m}$ showing superior temperature dependence and a maximum operating temperature of $280 \mathrm{~K}$. The triangles are for a $\mathrm{AlGa} / \mathrm{GaAs}$ heterostructure with the greatest carrier confinement.

Figure 13 summarises the improvements in the temperature dependence of GaAs lasers using the arguments presented above. The device with the poorest thermal behaviour and the lowest operating temperature (200 K), emits at $\sim 9 \mu \mathrm{m}$ and is based on a 33\% concentration $\mathrm{Al}$ heterostructure. The highest operating temperature to date, $280 \mathrm{~K}$, has been achieved with a heterostructure of similar composition but a longer emission wavelength $(\sim 11 \mu \mathrm{m})$. The best temperature dependence has been achieved using an $\mathrm{AlGa} / \mathrm{GaAs}$ heterostructure with a $\Gamma-\Gamma$ band offset of $\sim 1 \mathrm{eV}$ ( $c f$. $295 \mathrm{meV}$ for $\mathrm{Al}=33 \%$ ). Although the maximum operating temperature is limited to $230 \mathrm{~K}$ by NDR effects, a device of this type is clearly a good candidate for room temperature operation.

\section{CONCLUSIONS}

To summarise, we have presented the results of an extensive research programme on GaAs based QC lasers. These devices have optimised low-loss Al-free waveguides which allow very high peak power, in excess of $1 \mathrm{~W}$ at $77 \mathrm{~K}$. The maximum 
temperature of operation of the lasers is $280 \mathrm{~K}$ and there is no physical limitation to hinder a further optimisation of their thermal characteristics, in order to reach and overcome room temperature. New devices made in AlAs/GaAs heterostructures with a specific design to suppress the thermal activated processes show very high $T_{0}$ $(=320 \mathrm{~K})$ and slope efficiency independent from the temperature.

\section{References}

[1] F. Capasso, MRS Bull. 16 (1991) 23-29.

[2] J. Faist, F. Capasso, D.L. Sivco, C. Sirtori, A.L. Hutchinson and A.Y. Cho, Sci 264 (1994) 553-556.

[3] J. Faist, F. Capasso, D.L. Sivco, A.L. Hutchinson and A.Y. Cho, Appl. Phys. Lett. 72 (1998) 680-682.

[4] A. Tredicucci, C. Gmachl, F. Capasso, A.L. Hutchinson, D.L. Sivco and A.Y. Cho, Appl. Phys. Lett. 76 (2000) 2164-2166.

[5] C. Sirtori, J. Faist, F. Capasso, D.L. Sivco, A.L. Hutchinson and A.Y. Cho, IEEE Photon. Technol. Lett. 9 (1997) 294-296.

[6] J. Faist, F. Capasso, C. Sirtori, D.L. Sivco, J.N. Baillargeon, A.L. Hutchinson and A.Y. Cho, Appl. Phys. Lett. 68 (1996) 3680-3682.

[7] C. Sirtori, P. Kruck, S. Barbieri, P. Collot, J. Nagle, M. Beck, J. Faist and U. Oesterle, Appl. Phys. Lett. 73 (1998) 3486-3488.

[8] A. Yariv, Quantum Electronics, $3^{\text {rd }}$ Ed. (Wiley, New York, 1989).

[9] J.-C. Garcia, E. Rosencher, Ph. Collot, N. Laurent, J.L. Guyaux, B. Vinter and J. Nagle, Appl. Phys. Lett. 71 (1997) 3752-3754.

[10] C. Gmachl, F. Capasso, A. Tredicucci, D.L. Sivco, A.L. Hutchinson, S.N.G. Chu and A. Y. Cho, Appl. Phys. Lett. 73 (1998) 3830-3832.

[11] J. Faist, A. Tredicucci, F. Capasso, C. Sirtori, D.L. Sivco, J.N. Baillargeon, A.L. Hutchinson and A.Y. Cho, IEEE J. Quantum Electron. 34 (1998) 336-343.

[12] B.A. Puldus, T.G. Spence, R.N. Zare, J. Oomens, F.J.M. Harren, D.H. Parker, C. Gmachl, F. Capasso, D.L. Sivco, J.N. Baillargeon, A.L. Hutchinson and A.Y. Cho, Optics Lett. 24 (1999) 178-180.

[13] S.W. Sharpe, J.F. Kelly, J.S. Hartman, Gmachl, F. Capasso, D.L. Sivco, J.N. Baillargeon and A.Y. Cho, Optics Lett. 23 (1998) 1396-1398.

[14] N. Chand, T. Henderson, J. Klem, W.T. Messelink, R. Fisher, Y.-C. Chang and H. Morkoç, Phys. Rev. B 30 (1984) 4481-4492.

[15] C. Sirtori, F. Capasso, J. Faist, A.L. Hutchinson, D.L. Sivco and A.Y. Cho, IEEE J. Quantum Electron. 34 (1998) 1722-1729.

[16] R. Ferreira and G. Bastard, Phys. Rev. B 40 (1989) 1074-1086.

[17] A. Tredicucci, C. Gmachl, F. Capasso, D.L. Sivco, A.L. Hutchinson and A.Y. Cho, Appl. Phys. Lett. 74 (1998) 638-640. 
[18] C. Sirtori, C, Gmachl, F. Capasso, J. Faist, D.L. Sivco, A.L. Hutchinson and A.Y. Cho, Optics Lett. 23 (1998) 1366-1368.

[19] W.W. Bewley, C.L. Felix, E.H. Aifer, I. Vurgaftman, L.J. Olafsen, J.R. Meyer, H. Lee, R.U. Martinelli, J.C. Connolly, A.R. Sugg, G.H. Olsen, M.J. Yang, B.R. Bennett and B.V. Shanabrook, Appl. Phys. Lett. 73 (1998) 3833-3835.

[20] C. Sirtori, P. Kruck, S. Barbieri, H. Page, P. Collot, J. Nagle, M. Beck, J. Faist and U. Oesterle, Appl. Phys. Lett. 75 (1999) 3911-3913.

[21] C. Sirtori, S. Barbieri, P. Kruck, V. Piazza, M. Beck, J. Faist, U. Oesterle and J. Nagle, IEEE Photonics Technol. Lett. 11 (1999) 1090-1092.

[22] C. Sirtori, J. Faist, F. Capasso, D.L. Sivco, A.L. Hutchinson and A.Y. Cho, Appl. Phys. Lett. 66 (1996) 3242-3244.

[23] B. Jensen, in Handbook of Optical Constants, edited by E.D. Palik (Academic, Orlando, 1985), Chapter 9.

[24] B.W. Hakki and T.L. Paoli, J. Appl. Phys. 46 (1975) 1299-1306.

[25] W. Schrenk, N. Finger, S. Gianordoli, L. Hvozdara, G. Strasser and E. Gornik, Appl. Phys. Lett. 76 (2000) 253-255.

[26] L. Hvozdara, A. Lugstein, S. Gianordoli, W. Schrenk, G. Strasser, K. Unterrainer, E. Bertgnolli and E. Gornik, Appl. Phys. Lett. 77 (2000) 1077-1079.

[27] S. Gianordoli , L. Hvozdara, G. Strasser, W. Schrenk, J. Faist and E. Gornik, IEEE J. Quantum Electron. 36 (2000) 458-464.

[28] Gmachl, A.M. Sergent, A. Tredicucci, F. Capasso, A.L. Hutchinson, D.L. Sivco, J.N. Baillargeon, S.N.G. Chu and A.Y. Cho, IEEE Photon. Technol. Lett. 11 (1999) 1369-1371.

[29] P. Kruck, H. Page, C. Sirtori, S. Barbieri, M. Stellmacher and J. Nagle, Appl. Phys. Lett. 76 (2000) 3340-3342.

[30] S. Barbieri, C. Sirtori, H. Page, M. Beck, J. Faist and J. Nagle, IEEE J. Quantum Electron. 36 (2000) 736-741.

[31] S. Barbieri, C. Sirtori, H. Page, M. Stellmacher and J. Nagle, Design Strategies for GaAs-Based Unipolar Lasers: Optimum Injector-Active Region Coupling Via Resonant Tunneling, Appl. Phys. Lett. 78 (2001) 282.

[32] C. Becker, C. Sirtori, H. Page, G. Glastre, V. Ortiz, X. Marcadet, M. Stellmacher and J. Nagle, Appl. Phys. Lett. 77 (2000) 463-465.

[33] The manifold of states II arises from the coupling of the excited states of the quantum wells forming the injector. The height of the AlAs barriers allows to increase the confinement of these states and therefore to strongly enhance the energy difference $\Delta E_{\text {act }}$, which controls the activated processes, see Figure 10.

[34] K. Huang and B. Zhu, Phys. Rev. B 38 (1988) 13377-13386. 